\title{
Comparing the Effect of Metformin and Acarbose Accompanying Clomiphene on the Successful Ovulation Induction in Infertile Women with Polycystic Ovary Syndrome
}

\author{
Masomeh Rezai ${ }^{1}$, Mohmmad Jamshidi ${ }^{2}$, Robabeh Mohammadbeigi ${ }^{3}$, Fariba Seyedoshohadaei ${ }^{1}$, Somaye \\ Mohammadipour $^{1} \&$ Ghobad Moradi $^{4}$ \\ ${ }^{1}$ Department of Obstetrics \& Gynecology, Faculty of Medicine, Kurdistan University of Medical Sciences, \\ Sanandaj, Iran \\ ${ }^{2}$ Cellular and Molecular Research Center, Zahedan University of Medical Sciences, Zahedan, Iran \\ ${ }^{3}$ Department of Obstetrics \& Gynecology, Faculty of Medicine, Iran University of Medical Sciences, Tehran, \\ Iran \\ ${ }^{4}$ Social Determinants of Health Research Center, Kurdistan University of Medical Sciences, Sanandaj, Iran \\ Correspondence: Somaye Mohammadipour, Department of Obstetrics \& Gynecology, Faculty of Medicnie, \\ Kurdistan University of Medical Sciences, Sanandaj, Iran. Tel: 98-871-3366-0089. E-mail: \\ mohamadjamshidi87@yahoo.com
}

Received: October 31, 2015 Accepted: January 5, 2016 Online Published: January 31, 2016

doi:10.5539/gjhs.v8n9p281 URL: http://dx.doi.org/10.5539/gjhs.v8n9p281

\begin{abstract}
The aim of this study was to compare the effects of Metformin and Acarbose accompanying Clomiphene on the successful ovulation induction in infertile women with polycystic ovary syndrome. This randomized double blind clinical trial study was performed on 60 women with polycystic ovary syndrome. Women were selected and randomly divided in two control and intervention groups. Intervention group received Acarbose $100 \mathrm{mg} /$ day for 3 months. In the first, second, and third weeks, they received 1 tablet, 2 tablets, and 3 tablets per day respectively. In addition, they received $100 \mathrm{mg}$ Clomiphene from third to seventh day of menstruation, during the 3 month treatment period. The control group received Metformin $500 \mathrm{mg} /$ day for 3 months. In the first, second, and third weeks, they received 1 tablet, 2 tablets, and 3 tablets per day respectively. In addition, they received $100 \mathrm{mg}$ Clomiphene from third to seventh day of menstruation, during the 3 month treatment period. All the subjects in both groups before and after the treatment were examined for hirsutism, acne, oral glucose tolerance test, serum triglycerides, cholesterol, LDL, HDL. Also, induction of ovulation was assessed by vaginal ultrasound. The Mean of BMI and fasting glucose tolerance test in Acarbose group was less than Metformin group $(\mathrm{P}=0.05)$. The mean of triglycerides, LDL and HDL levels did not differ between the two groups after the intervention (P > 0.05). The mean of cholesterol levels were different in the two groups after the intervention $(P=0.04)$. Frequency of ovulation induction in those who received Acarbose $(78.5 \%)$ was more than those who received Metformin (46.6) $(\mathrm{P}=0.012)$. Comparing with Metformin, Acarbose accompanying Clomiphene was more effective in ovulation induction and decreasing body mass index in infertile women with polycystic ovary syndrome.
\end{abstract}

Keywords: acarbose, infertility, metformin, ovulation induction, Polycystic Ovary syndrome

\section{Introduction}

Polycystic Ovary syndrome (PCOS) is a heterogeneous endocrine disorder. One out of every 15 women (approximately $5-10 \%$ of women) is suffering from it globally. The basic disorder in this syndrome is increasing androgen secretion and in many patients it is due to abnormal insulin activity. The cause of this syndrome is unknown, but genetic studies considered the impact of prenatal environment, lifestyle or both (Sonez, 2005). Most women with PCOS and infertility are insulin resistant. High secretion of insulin may stimulate ovarian androgen secretion and a decrease in insulin production may decrease ovarian androgen secretion and increase ovulation rate (Galluzzo, Amato, \& Giordano, 2008).

Some studies have shown that the combination of Metformin and Clomiphene for ovulation induction in women 
with PCOS is more effective than Clomiphene alone (Leanza et al., 2014) other studies have also shown that in women, who received Metformin, pregnancy rate was higher and abortion rate was lower (Moll et al., 2008). However, Moll and Legro studies showed that adding Metformin to Clomiphene did not increase the live birth rate (Moll et al., 2008; Legro et al., 2007). Acarbose is a glucosidase inhibitor, which is used in the treatment of type 2 diabetes and is also under study in Clomiphene-resistant patients (Karimzadeh \& Javedani, 2010). Other studies have shown that Acarbose improve hirsutism, acne, and menstrual irregularities through a reduction in the increasing levels of androgens and androgen band. In comparison, Acarbose reduces more weight and improve menstrual irregularities and signs of fertility in Clomiphene-resistant women with PCOS. In obese women with PCOS using Acarbose, symptoms of cardiovascular risk were decreased significantly after 6 months of treatment. Also, gastrointestinal complications in patients who took Acarbose are less than patients who took Metformin (Kircher \& Smith, 2008).

Given the contradiction of the effects of Acarbose compared with Metformin on the clinical symptoms and induced ovulation in women with polycystic ovary, the aim of this study was to compare the effects of Metformin and Acarbose accompanying Clomiphene on the successful ovulation induction in infertile women due to polycystic ovary syndrome.

\section{Method}

This double-blind clinical trial study was conducted on infertile women with polycystic ovary syndrome who referred to Besat Hospital clinic, Sanandaj, Iran in 2014. The Inclusion criteria were; 20 to 40 years of age, Rotterdam standard diagnostic criteria A: oligomenorrhea or amenorrhea, B: clinical findings or biochemical hyperandrogenism and C: polycystic ovaries on ultrasound. Exclusion criteria were: smoking, Cushing's syndrome, thyroid dysfunction, tumors that generate androgens; patients with liver, kidney and heart disease, diabetes; treatment with other drugs of polycystic ovary syndrome such as Aldactone, Diane and Clomiphene and finally Clomiphene- resistant patients. The sample size was 60 patients who randomly divided into intervention and control groups using 4 blocking methods. This study was approved by the Ethics Committee of Kurdistan University of Medical Sciences and has been registered in the Iranian Registry of Clinical Trials with registration number IRCT2014092912789N7.

Infertile women who were willing to participate in the study and had inclusion criteria were enrolled in the study. Written consent was obtained from the participants. The Intervention group received Acarbose $100 \mathrm{mg} /$ day for 3 months. In the first, second, and third weeks, they received 1 tablet, 2 tablets, and 3 tablets per day respectively. In addition, they received a daily dose of $100 \mathrm{mg}$ Clomiphene for 3 months during the treatment period. The control group received Metformin $500 \mathrm{mg} /$ day for 3 months. In the first, second, and third weeks, they received 1 tablet, 2 tablets, and 3 tablets per day respectively. Also, they received $100 \mathrm{mg}$ Clomiphene daily during the 3 months treatment period. All the subjects in both groups before and after the treatment were examined for hirsutism, acne, oral glucose tolerance test, serum triglycerides, cholesterol, LDL and HDL. Also, induction of ovulation was assessed using vaginal ultrasound.

A gynecology resident followed up the patients during the course of treatment for drug usage and possible side effects, including gastrointestinal side effects such as bloating and diarrhea. For blinding purposes, Acarbose and Metformin were given to patients in the same packages. Also, drug packages were designed as A and B and a person who gave the drugs to the patients were unaware of the contents of the packages.

Data of 58 women participated in the study were entered into SPSS version 18 (2 patients in Acarbose group withdrew from the study due to diarrhea and gastritis). Descriptive statistics (frequency, mean and standard deviation) as well as chi-square, t-test and Fisher's test were used to analyze the data.

\section{Results}

Results of this study showed that the mean age of Metformin group was $4.7 \pm 26.3$ and in Acarbose group it was $4.6 \pm 26.3$ years. In terms of education, $75 \%$ of Acarbose and $70.5 \%$ of Metformin group had a high school diploma or less. $80 \%$ of Acarbose group and $86.6 \%$ of Metformin group were housewives. $30 \%$ of the Metformin group and $21.55 \%$ of Acarbose group were from rural areas.

Duration of infertility and PCOS in Metformin group was $2.66 \pm 1.66$ years and $47.2 \pm 31.9$ months and in Acarbose group they were $3.67 \pm 6.52$ years and $44.6 \pm 22.1$ months respectively. In terms of previous treatment history, $50 \%$ of the Acarbose group and $70 \%$ of Metformin group had a history of treatment.

Mean of BMI and fasting glucose tolerance test in Acarbose group was less than Metformin group $(\mathrm{P}=0.05)$. The mean of triglycerides, LDL and HDL levels did not differ between the two groups after the intervention (P > 0.05). The mean of cholesterol levels were different in the two groups after the intervention $(\mathrm{P}=0.04)$ (Table 1). 
Frequency of ovulation induction in those who received Acarbose (78.5\%) was more than those who received Metformin (46.6) $(\mathrm{P}=0.012)$. Frequency of hirsutism and acne in two groups were not different (Table 2), also frequency of gastrointestinal side effects was similar in both groups $(\mathrm{P}>0.05)$ (Table 3$)$.

Table 1. Comparison of the clinical and laboratory findings before and after treatment

\begin{tabular}{|c|c|c|c|c|c|c|}
\hline \multirow{2}{*}{ Clinical Tests } & \multicolumn{3}{|c|}{ Before } & \multicolumn{3}{|c|}{ After } \\
\hline & Acarbose & Metformin & P Value & Acarbose & Metformin & P Value \\
\hline Fasting glucose tolerance test & $88.2 \pm 16.05$ & $92.1 \pm 16.3$ & 0.37 & $83.3 \pm 10.7$ & $93.6 \pm 19.3$ & 0.024 \\
\hline glucose tolerance test 2 hours after & $125.6 \pm 25.9$ & $129.7 \pm 24.2$ & 0.61 & $122 \pm 15.6$ & $127.3 \pm 16.2$ & 0.23 \\
\hline Triglycerides & $122.8 \pm 42.3$ & $128.2 \pm 47.7$ & 0.65 & $118.1 \pm 36.5$ & $123.5 \pm 31$ & 0.56 \\
\hline Cholesterol & $173.6 \pm 28.6$ & $183.9 \pm 25.5$ & 0.15 & $162.2 \pm 36.5$ & $176.9 \pm 31$ & 0.04 \\
\hline LDL & $100.4 \pm 27.8$ & $109.2 \pm 24.6$ & 0.21 & $93.3 \pm 20.3$ & $103.6 \pm 26.5$ & 0.12 \\
\hline HDL & $45.4 \pm 5.7$ & $46.3 \pm 12.9$ & 0.74 & $44.3 \pm 7.6$ & $47.4 \pm 12.2$ & 0.27 \\
\hline BMI & $26.9 \pm 1.8$ & $27.3 \pm 2.4$ & 0.28 & $25.9 \pm 1.9$ & $27.2 \pm 2.4$ & 0.04 \\
\hline
\end{tabular}

Results are expressed as mean $\pm \mathrm{SD}$.

Table 2. Comparison of the clinical findings before and after the treatment

\begin{tabular}{|c|c|c|c|c|c|c|}
\hline & \multicolumn{3}{|l|}{ Before } & \multicolumn{3}{|l|}{ After } \\
\hline & Acarbose & Metformin & $\mathrm{P}$ & Acarbose & Metformin & $P$ \\
\hline ovulation induction & & & & & & 0.012 \\
\hline Yes & 0 & 0 & & $22(78.5)$ & $14(46.6)$ & \\
\hline No & $28(100)$ & $30(100)$ & & $6(22.5)$ & $16(53.4)$ & \\
\hline Acne & & & & & & 0.11 \\
\hline Yes & $9(32)$ & $8(36.7)$ & 0.64 & 0 & $2(25)$ & \\
\hline No & $19(68)$ & $22(73.3)$ & & $9(100)$ & $6(75)$ & \\
\hline Menstrual disorder & & & & & & 0.15 \\
\hline Yes & $23(82.1)$ & $24(80)$ & 0.83 & $17(73.9)$ & $13(54.1)$ & \\
\hline No & $5(17.9)$ & $6(20)$ & & $6(26.1)$ & $11(45.9)$ & \\
\hline Hirsutism & & & & & & 0.99 \\
\hline Yes & $14(50)$ & $14(46.6)$ & 0.8 & $2(14.2)$ & $2(12.2)$ & \\
\hline No & $14(50)$ & $16(53.4)$ & & $12(85.8)$ & $12(85.8)$ & \\
\hline
\end{tabular}

Data are No. $(\%)$.

Table 3. Comparison of the side effects in both groups

\begin{tabular}{lllll}
\hline Side Effects & $\begin{array}{l}\text { Group } \\
\text { Metformin }\end{array}$ & Acarbose & & \\
\hline Distension & & & P Value \\
Yes & $7(23 / 3)$ & $8(28 / 6)$ & 0.2 & \\
No & $23(76 / 7)$ & $20(71 / 4)$ & & \\
\hline Vomiting & & & 0.65 \\
Yes & 0 & $2(7 / 1)$ & 2.2 & \\
No & $30(100)$ & $26(92 / 9)$ & & 0.88 \\
\hline Diarrhea & & & \\
\hline
\end{tabular}




\begin{tabular}{lllll}
\hline Yes & $8(26.7)$ & $7(25)$ & 0.02 & \\
No & $22(73.3)$ & $21(75)$ & & \\
\hline Anorexia & & & 0.65 \\
Yes & $8(26.7)$ & $9(32.1)$ & 0.21 & \\
No & $22(73.3)$ & $19(67.9)$ & & \\
\hline
\end{tabular}

\section{Discussion}

In recent years, oral anti-diabetic drugs such as Metformin, Troglitazone, Rositroglitazone, and Acarbose are used for the treatment of patients with insulin resistance PCOS. Metformin is a biguanide antihyperglycemic that is widely used in patients with PCOS. Studies have shown the effect of Metformin in reducing insulin and improving the metabolic status of patients (Lord et al., 2003). Metformin side effects include anorexia, diarrhea, nausea, and vomiting suggesting the decrease in the use of this medication in patients with PCOS. In addition, an increase in blood lactic acid may be a life-threatening complication in diabetic patients (Lord et al., 2003; Jeffrey Chang, 2004) although these side effects have not been reported in women with PCOS (Legro, 2007).

In this study, the mean of BMI was changed from 26.9 to $25.9 \mathrm{~kg} / \mathrm{m}^{2}$ in Acarbose group and from 27.3 to 27.2 in the Metformin group. This showed a significant reduction of body mass index in Acarbose group. Weight loss in patients with type II diabetes after taking Acarbose has been reported in other studies (Chiasson et al., 2002). Kircher's systematic review has confirmed weight loss in women using Acarbose (Kircher, 2007). In Sönmez study, the mean of BMI in Acarbose group before treatment was $27.4 \mathrm{~kg} / \mathrm{m}^{2}$ and after treatment it was $26.3 \mathrm{~kg} / \mathrm{m}^{2}$. Mean of BMI in the Metformin group before and after treatment were 27.2 and 26.9, respectively, and there were significant differences between the two groups (Sonmez, 2005). In a study by Ciotta et al., Acarbose was used in 30 PCOS patients with normal BMI. In this group, there was no significant change in body mass index (Ciotta et al., 2001). In the same study, 61 women with PCOS were treated with Metformin and there were no significant changes in BMI (Nestler et al., 2002). In the meta-analysis conducted by Zhang et al. in 2014, to investigate the effects of Acarbose in patients with PCOS, there were no changes in the body mass index (Zhang, Hou, \& Zhao, 2014). Therefore; our findings are consistent with most of the above similar studies.

In the small intestine, Acarbose creates a dose-dependent reversible bond with alpha-glucosidase position in Oligosaccharides that reduces oligosaccharides and disaccharides hydrolysis, leading to reduced absorption of monosaccharide in the blood stream. Acarbose reduces $20 \%$ after meal blood sugar and increases glucose uptake, this will prevent the Hyperinsulinemia. This drug, through reducing absorption of glucose, leads to an indirect increase in Glucagon-like peptide-1 and by affecting the satiety center of the brain reduces appetite and weight (Qiao et al., 2015).

The prevalence of hirsutism before and after the treatment was not significantly different in the two groups and improved patients were similar in both groups. In the meta-analysis conducted by Zhang et al in 2014, the Galway Freeman criteria were not changed (Zhang, Hou, \& Zhao, 2014). Ciotta showed favorable impression of Acarbose on Freeman Galway criteria (Ciotta et al., 2001). In a study by Penna, Galway Freeman criteria in Acarbose group were lower than the control group. Given that incidence of hirsutism in the control group was higher before treatment, this difference was not necessarily related to the effects of Acarbose (Penna, 2005). Kircher approved hirsutism improvement in women using Acarbose (Kircher, 2008). Because of the inverse relationship between weight and sex hormone binding globulin, Acarbose increases this globulin through weight loss and increases insulin sensitivity and decreases LH and hyperandrogenism (Ciotta et al., 2001). Probably the cause of this difference is related to the duration of the intervention or treatment with Acarbose. Given the short period of 3 months of treatment in this study and long-term treatment of hirsutism, failure in treatment is logical.

In the present study, the mean of fasting glucose tolerance test was reduced after the intervention in Acarbose group than before, and had significant difference with Metformin group. In some studies Metformin $300 \mathrm{mg}$ daily with Acarbose 1.5 gr daily in PCOS patients, regardless of the weight was investigated and metabolic parameters were similar (Sonmez, 2005; Willson, 2008; Barbeiri \& Ehrmann, 2010; Ehrman, 2005; Azziz, 2009). Hanjalic-Beck showed that only in patients who had been treated with Metformin, levels of fasting insulin reduced significantly (Hanjalic-Beck et al., 2010). Ciotta showed desired effects of Acarbose on insulin sensitivity improvement (Ciotta, 2001). A study showed that Acarbose did not cause hypoglycemia or lactic acidosis (Guilherme et al., 2008) .This approach strengthened using a combination of these two drugs.

In both groups lipid profile reduced after the intervention compared to the before, but cholesterol level in 
Acarbose group was significantly lower than Metformin group. In Hanjalic-Beck study, patients who had been treated with Metformin showed significant reduction in cholesterol levels (Hanjalic-Beck et al., 2010). In Zhang et al meta-analysis, they showed that Acarbose reduces triglycerides and LDL and increases HDL compared with placebo (Zhang, Hou, \& Zhao, 2014). The results showed that improvement in menstrual disorders of Acarbose group (73.9\%) was greater than Metformin group (54.1\%). Other studies, including Hanjalic and Beck study the prevalence of menstrual regulation in the Metformin group were $70 \%$ and in Acarbose it was $78 \%$ (Hanjalic-Beck et al., 2010). Penna's study showed that in patients who received Acarbose compared to the control group, they had 2.67 times regular menstrual cycles (Penna, 2005). In Ciotta's study, $60 \%$ of patients in Acarbose group had regular menstrual periods (Ciotta, 2001). Kircher's systematic review confirmed menstrual cycle improvement in women who used Acarbose (Kricher, 2008). In Zhang's study, there was no significant difference between Acarbose and Metformin regarding menstrual pattern (Zhang, Hou, \& Zhao, 2014). The results of most of the studies are consistent with our findings. It seems that menstrual regulation is associated with weight loss, secretion of SHBG, free androgen reduction, and performance of ovarian hormones (Penna, 2005).

In our study, there was no difference between the two groups regarding acne treatment. But, Metformin group was better than Acarbose. Ciotta showed favorable effects of Acarbose on acne (Ciotta, 2001). In his systematic review, Kricher showed a positive effect of Acarbose on acne (Kricher, 2008).

Frequency of ovulation induction in Acarbose group (78.5\%) was significantly higher than Metformin group (46.6\%). In Ciotta's study, there was not a method to determine the ovulation rate during treatment with Acarbose (Ciotta, 2001). In Zhang et al meta-analysis, there was no significant difference between Acarbose and Metformin groups regarding increases in the ovulation rate (Zhang, Hou, \& Zhao, 2014). Sönmez et al showed that the ovulation rate increased in both Acarbose and Metformin groups and indicated that administration of Metformin improved follicle response to gonadotropins (Snomez, 2005). In 2010 Hanjalic-Beck studied the effects of Metformin versus Acarbose on ovulation rate. They studied the impact of hormones and metabolism in patients with polycystic ovary syndrome (PCOS). The ovulation rate in the Metformin group was $73 \%$ and in Acarbose was it was 59\%, but the difference was not statistically significant (Hanjalic-Beck et al., 2010).

Frequency of gastrointestinal side effects, including distension, vomiting, diarrhea and anorexia in both groups (about 28\%) was the same. In Zhang et al study, Acarbose caused side effects such as abdominal pain and diarrhea $(O R=23.78)$ (Zhang, Hou, \& Zhao, 2014).In another study, mild complications were observed in $84 \%$ of the patients in Acarbose group (Penna, 2005). In Hanjalic study, in Acarbose group (38\%) distension and diarrhea was less compared with Metformin group (80\%) (Hanjalic-Beck et al., 2010). Acarbose intestinal absorption rate was less than Metformin $(1.7 \%-5.0 \%)$ and this property reduced the dose dependent side effects such as distension and diarrhea. These side effects were minimized with Acarbose gradual increase. The most important Metformin side effects were nausea, vomiting, fatigue, diarrhea, particularly in patients with chronic renal failure. Also, Metformin reduced the intestinal absorption of vitamin B12.Due to the incidence of side effects, choosing the proper treatment method could be a limiting factor; therefore, Acarbose appears to be safer and more effective in the treatment of PCOS (Ciotta, 2001).

Penna et al study suggested using a combination of drugs for patients with PCOS, particularly in obese patients that standard treatments are less effective (Penna, 2005). Although there are a lot about clinical, metabolic, and reproductive benefits of Metformin; however, stabilizing the controlled studies showed that the results were moderate (Harborne et al., 2003). This has opened a new horizon in the combination therapy.

In conclusion, regarding ovulation induction and reduction of body mass index of women with PCOS, usage of Acarbose with Clomiphene is more effective than Metformin. Also Acarbose reduces the lipid profile better than Metformin; though, they have the same gastrointestinal side effects.

\section{Acknowledgments}

We the authors wish to thank Vice Chancellor for Research, Kurdistan University of Medical Sciences to support this study financially.

\section{Conflict of Interest}

The authors declare that there is no conflict of interests regarding the publication of this paper.

\section{References}

Azziz, R., Carmina, E., Dewailly, D., Diamanti-Kandarakis, E., Escobar-Morreale, H., Futterweit, W., ... Witchel, S. (2009). The Androgen Excess and PCOS Society criteria for the polycystic ovary syndrome: the complete task force report. Fertility and Sterility, 91(2), 456-488. PMID: 18950759. 
http://dx.doi.org/10.1016/j.fertnstert.2008.06.035

Barbeiri, R. L., \& Ehrmann, D. A. (2010). Clinical manifestations of polycystic ovary syndrome in adults. Retrieved August 15 ${ }^{\text {th }}$, 2015, from http://www.uptodate.com/home/index.html

Chiasson, J., Josse, R., Gomis, R., Hanefeld, M., Karasik, A., \& Laakso, M. (2002). Acarbose for prevention of type 2 diabetes mellitus: the STOP-NIDDM randomised trial. The Lancet, 359(9323), 2072-2077. http://dx.doi.org/10.1016/S0140-6736(02)08905-5

Ciotta, L. (2001). Clinical, endocrine and metabolic effects of acarbose, an alpha-glucosidase inhibitor, in PCOS patients with increased insulin response and normal glucose tolerance Human Reproduction, 16(10), 2066-2072. PMID: 11574493. http://dx.doi.org/10.1093/humrep/16.10.2066

Ehrmann, D. (2005). Polycystic Ovary Syndrome. New England Journal of Medicine, 352(12), 1223-1236. PMID: 15788499. http://dx.doi.org/10.1056/NEJMra041536

Galluzzo, A., Amato, M., \& Giordano, C. (2008). Insulin resistance and polycystic ovary syndrome. Nutrition, Metabolism and Cardiovascular Diseases, $18(7), \quad$ pp.511-518. http://dx.doi.org/10.1016/j.numecd.2008.05.004

Guilherme, A., Virbasius, J., Puri, V., \& Czech, M. (2008). Adipocyte dysfunctions linking obesity to insulin resistance and type 2 diabetes. Nature Reviews Molecular Cell Biology, 9(5), 367-377. http://dx.doi.org/10.1038/nrm2391

Hanjalic-Beck, A., Gabriel, B., Schaefer, W., Zahradnik, H., Schories, M., Tempfer, C., Keck, C., \& Denschlag, D. (2010). Metformin versus acarbose therapy in patients with polycystic ovary syndrome (PCOS): A prospective randomised double-blind study. Gynecological Endocrinology, 26(9), 690-697. PMID: 20626240. http://dx.doi.org/10.3109/09513591003686379

Harborne, L., Fleming, R., Lyall, H., Norman, J., \& Sattar, N. (2003). Descriptive review of the evidence for the use of metformin in polycystic ovary syndrome. The Lancet, 361(9372), 1894-1901. PMID: 12788588. http://dx.doi.org/10.1016/S0140-6736(03)13493-9

Jeffrey Chang, R. (2004). A practical approach to the diagnosis of polycystic ovary syndrome.American Journal of Obstetrics and Gynecology, 191(3), 713-717. PMID: 15467530. http://dx.doi.org/10.1016/j.ajog.2004.04.045

Karimzadeh, M., \& Javedani, M. (2010). An assessment of lifestyle modification versus medical treatment with clomiphene citrate, metformin, and clomiphene citrate-metformin in patients with polycystic ovary syndrome. Fertility and Sterility, 94(1), 216-220. http://dx.doi.org/10.1016/j.fertnstert.2009.02.078

Kircher, C., \& Smith, K. (2008). Acarbose for Polycystic Ovary Syndrome. Annals of Pharmacotherapy, 42(6), 847-851. PMID: 18460589. http://dx.doi.org/10.1345/aph.1K639

Leanza, V., Coco, L., Grasso, F., Leanza, G., Zarbo, G., \& Palumbo, M. (2014). vulation induction with clomiphene citrate and metformin in women with polycystic ovary syndrome. Minerva Ginecologica, 66(3), 299-301. PMID: 24971785.

Legro, R., Barnhart, H., Schlaff, W., Carr, B., Diamond, M., Carson, S., ... Myers, E. (2007). Clomiphene, Metformin, or Both for Infertility in the Polycystic Ovary Syndrome. New England Journal of Medicine, 356(6), 551-566. http://dx.doi.org/10.1056/NEJMoa063971 PMID: 17287476

Moll, E., Korevaar, J., Bossuyt, P., \& van der Veen, F. (2008). Does adding metformin to clomifene citrate lead to higher pregnancy rates in a subset of women with polycystic ovary syndrome?.Human Reproduction, 23(8), pp.1830-1834.http://dx.doi.org/10.1093/humrep/den182 PMID: 18487613

Nestler, J., Stovall, D., Akhter, N., Iuorno, M., \& Jakubowicz, D. (2002). Strategies for the use of insulin-sensitizing drugs to treat infertility in women with polycystic ovary syndrome. Fertility and Sterility, 77(2), 209-215. PMID: 11821072

Penna, I. (2005). Acarbose in obese patients with polycystic ovarian syndrome: a double-blind, randomized, placebo-controlled study. Human Reproduction, 20(9), 2396-2401. http://dx.doi.org/10.1093/humrep/dei104 PMID: 16006454.

Qiao, W., Li, J., Li, Y., Qian, D., Chen, L., Wei, X., Jin, J., \& Wang, Y. (2016). Acarbose, the $\alpha$-glucosidase inhibitor, attenuates the blood pressure and splanchnic blood flow responses to meal in elderly patients with postprandial hypotension concomitant with abnormal glucose metabolism. Blood Pressure Monitoring, 21(1), 38-42. http://dx.doi.org/10.1097/MBP.0000000000000160 
Sonmez, A. (2004). Comparison of the effects of acarbose and metformin use on ovulation rates in clomiphene citrate-resistant polycystic ovary syndrome. Human Reproduction, 20(1), 175-179. PMID: 15513975

Wilson, E. E. (2008). Polycystic ovarian syndrome and hyperandrogenism. In J. O. Schorge, (Ed.), Williams Gynecology. New York: McGraw-Hill Medical; 2008.

Zhang, Y., Hou, L., \& Zhao, T. (2014). Effects of Acarbose on Polycystic Ovary Syndrome: A Meta-analysis. Exp Clin Endocrinol Diabetes, 122(06), 373-378. PMID: 24941435. http://dx.doi.org/10.1055/s-0034-1375676

\section{Copyrights}

Copyright for this article is retained by the author(s), with first publication rights granted to the journal.

This is an open-access article distributed under the terms and conditions of the Creative Commons Attribution license (http://creativecommons.org/licenses/by/3.0/). 